\title{
Design and Expected Performance of GISMO-2, a Two Color Millimeter Camera for the IRAM $30 \mathrm{~m}$ Telescope
}

\author{
Johannes Staguhn • Dominic Benford • Eli Dwek • Gene Hilton • \\ Dale Fixsen • Kent Irwin • Christine Jhabvala • Attila Kovacs • \\ Samuel Leclercq • Stephen Maher • Tim Miller • S. Harvey Moseley • \\ Elmer Sharp · Edward Wollack
}

Received: 13 August 2013 / Accepted: 15 January 2014

(c) Springer Science+Business Media New York 2014

\begin{abstract}
We present the main design features for the GISMO-2 bolometer camera, which we build for background-limited operation at the IRAM $30 \mathrm{~m}$ telescope on Pico Veleta, Spain. GISMO-2 will operate simultaneously in the 1 and $2 \mathrm{~mm}$ atmospherical windows. The $1 \mathrm{~mm}$ channel uses a $32 \times 40$ TES-based backshort under grid (BUG) bolometer array, the $2 \mathrm{~mm}$ channel operates with a $16 \times 16$ BUG array. The camera utilizes almost the entire full field of view provided by the telescope. The optical design of GISMO-2 was strongly influenced by our experience with the GISMO $2 \mathrm{~mm}$ bolometer camera, which is successfully operating at the $30 \mathrm{~m}$ telescope. GISMO is accessible to the astronomical community through the regular IRAM call for proposals.
\end{abstract}

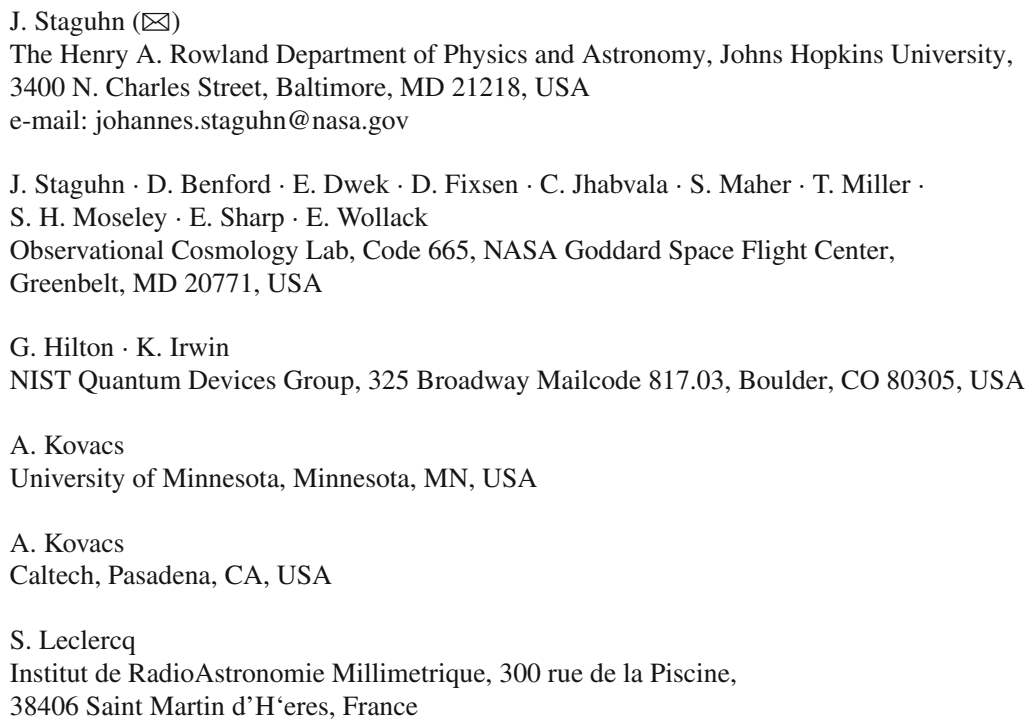




\section{Introduction}

Large arrays of superconducting transition edge sensor (TES) Bolometers by now have matured sufficiently to be available for background-limited (sub-)millimeter astronomy (e.g. [1]). Our team has a long legacy in building and using TES based instrumentation, having successfully fielded FIBRE, the first TES based instrument with multiplexed readout [2], and recently the Goddard-IRAM Superconducting $2 \mathrm{~mm}$ Observer GISMO [3], which is available for astronomical observations (e.g. [4,5]) at the IRAM $30 \mathrm{~m}$ telescope in Spain through the regular IRAM call for proposals. GISMO uses an $8 \times 16$ array of close-packed, high sensitivity, TES bolometers with a pixel size of $(2 \times 2) \mathrm{mm}^{2}$, which was built in the Detector Development Laboratory (DDL) at NASA/GSFC. The detector bolometer array architecture in GISMO is based on our backshort under grid (BUG) design, which enables kilopixel size arrays for future ground based, suborbital, and space based X-ray and far-infrared through millimeter cameras [6]. Its wide field refractive optics allow for a compact design of the instrument. This design is similar to the design used in the Atacama Cosmology Telescope's camera ACT, and the $3 \mathrm{~mm}$ Mustang camera at the GBT, both of which were built with the involvement of our group and which use superconducting arrays also provided by NASA/GSFC. With an achieved NEFD of $10 \mathrm{mJy} \mathrm{s}^{1 / 2}$, GISMO's performance is near- background limited.

Building on the heritage established by GISMO, and using the lessons learned as guidelines for improving the performance, we have developed the concept for a 2-color kilopixel millimeter bolometer camera, GISMO-2. In order to allow for background-limited observations, the required sensitivity expressed in noise equivalent power (NEP) for GISMO-2's detectors in the $2 \mathrm{~mm}$ atmospheric window at Pico Veleta is approximately $4 \times 10^{-17} \mathrm{~W} / \sqrt{ } \mathrm{Hz}$. At $1.1 \mathrm{~mm}$ the required NEP is about a factor of two higher. Our GISMO detectors have achieved the required $2 \mathrm{~mm}$ sensitivity even with a transition temperature Tc $=459 \mathrm{mK}$. However, the TES bolometer arrays in GISMO-2 will operate at a significantly lower transition temperature than the GISMO detectors (Tc $\sim 200 \mathrm{mK}$ ), therefore providing for a larger dynamic range. This will improve the 1/f noise stability over the one in GISMO (which has a $0.1 \mathrm{~Hz}$ 1/f knee). This will in particular allow for the recovery of large-scale emission obtained with wide observing patterns. With straylight issues being one of the major obstacles in the design of large bolometer cameras, the instrument will have an improved straylight omission over the GISMO optical design by incorporation of both a cold pupil and field stop and by using a new improved (sub-) millimeter black paint.

\section{Scientific Objectives and Motivation}

GISMO-2 is a camera designed for the low sky background of the 1 and $2 \mathrm{~mm}$ spectral bands with the major goal of obtaining large deep blank sky galaxy surveys. Due to the strong negative K-correction at millimeter wavelengths, these surveys will in particular discover a large number of very high-redshift dusty galaxies $(z>4)$. The resulting science will be complementary e.g. to high-redshift science obtained e.g. by 


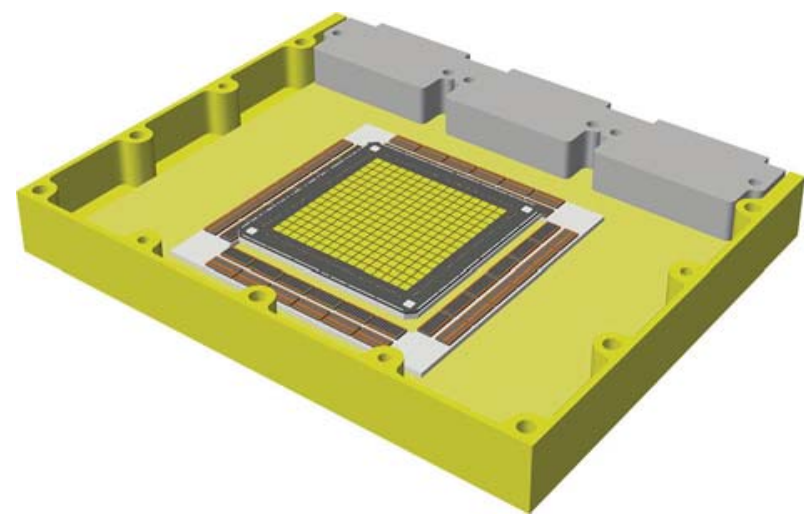

Fig. 1 The $2 \mathrm{~mm}, 16 \times 16$ pixel GISMO-2 array with $2 \mathrm{~mm}$ pixel pitch is read out by 1 -dimensional SQUID multiplexers from NIST/Boulder (arranged around the array) (Color figure online)

ALMA, since interferometers are not well suited to obtain large field surveys. For a more detailed motivation for large millimeter surveys, see [7].

\section{Technical Description}

\subsection{Detectors}

GISMO-2 is enabled by a detector technology developed at the NASA/Goddard Space Flight Center. We have an ongoing, multi-year program to develop key technologies necessary to build kilopixel arrays with the BUG architecture. The arrays consist of TES detectors with resonant backshorts. Each bolometer array is a filled squaregrid of micro-machined bolometers on $1.5 \mu \mathrm{m}$ SOI silicon, having superconducting transition edge sensors and infrared absorbing films. The absorber is bismuth, protected with a layer of silicon monoxide, to slow the adsorption of atmospheric water vapor into the bismuth over time. To optimize the optical efficiency, a $\lambda / 4$ backshort is placed behind the detectors. The $16 \times 16$ pixel $2 \mathrm{~mm}$ array on GISMO-2 (Fig. 1) utilizes these two components and is read out in the plane of the detector by 1dimensional NIST SQUID multiplexers. The kilopixel $1 \mathrm{~mm}$ array has a more complex architecture: 2-dimensional SQUID Multiplexers, also provided by NIST/Boulder, are bump-bonded to the TES array with the integrated backshorts. The process of bump-bonding this final device is called "hybridization". A fully hybridized $32 \times 40$ detector array (Fig. 2) has been produced at Goddard and is in the process of being characterized.

\subsubsection{Cryocooler:}

GISMO's dry cryogenic system is designed as a straightforward, simple to operate system with no cryogenic moving parts. Cooling power from 300 to $3 \mathrm{~K}$ is provided by a closed-cycle Cryomech PT415 cryo-cooler. A combination of ${ }^{4} \mathrm{He}$ and ${ }^{3} \mathrm{He}$ 


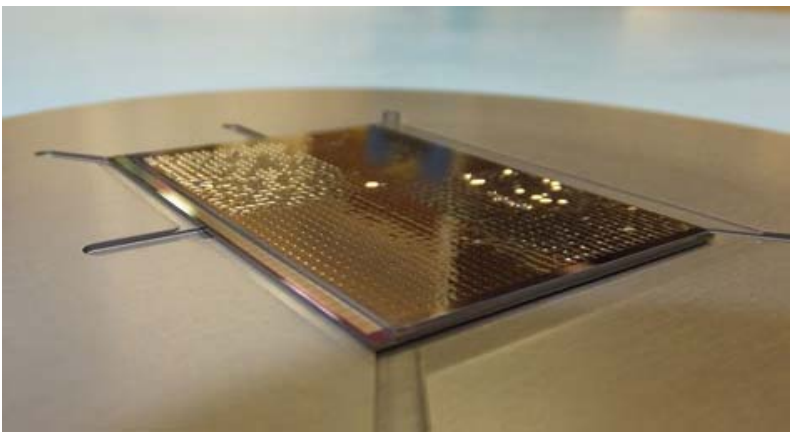

Fig. 2 A hybridized $1 \mathrm{~mm}, 32 \times 40$ pixel prototype BUG array with integrated backshort and bump-bonded to a 2-dimensional SQUID multiplexer from NIST/Boulder (Color figure online)

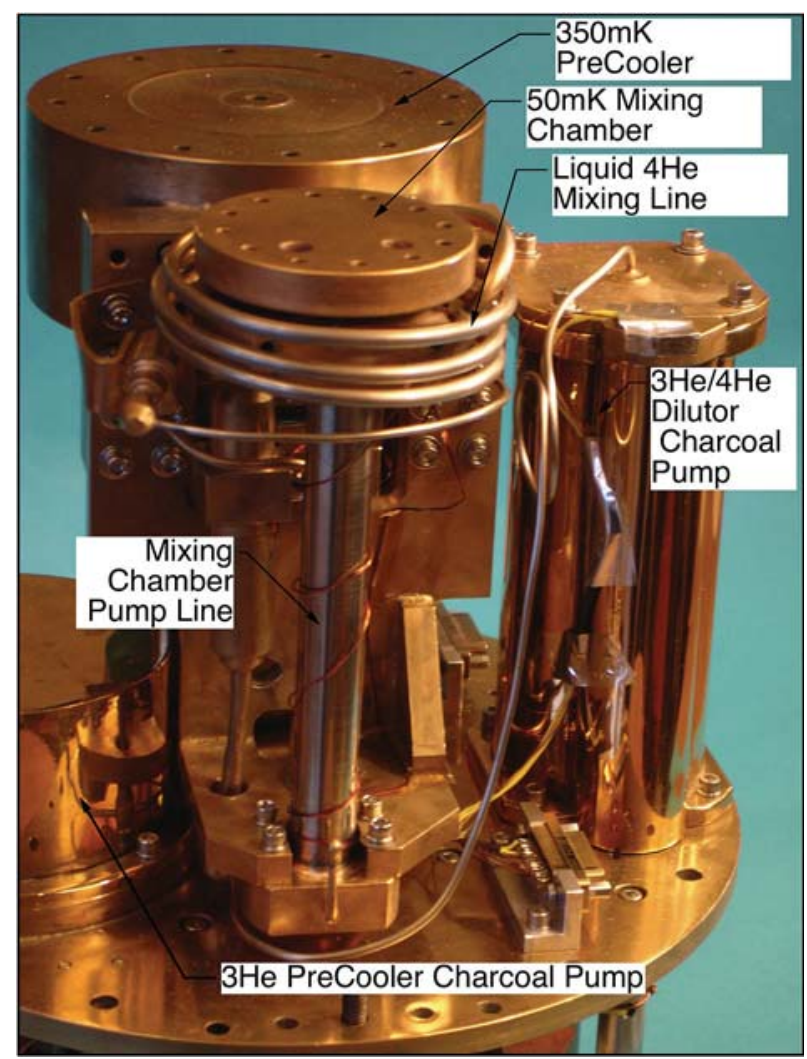

Fig. 3 The GISMO-2 fridge consists of a ${ }^{3} \mathrm{He} /{ }^{4} \mathrm{He}$ dilution refrigerator, which is buffered by a ${ }^{4} \mathrm{He} /{ }^{3} \mathrm{He}$ adsorption pre-cooler (Color figure online)

evaporation coolers, produced by Chase Research Cryogenics, then pre-cool a dilution refrigerator (Fig. 3) which provides a base temperature of $50 \mathrm{mK}$ for the detector arrays with a hold time of close to $24 \mathrm{~h}$.

\section{Springer}




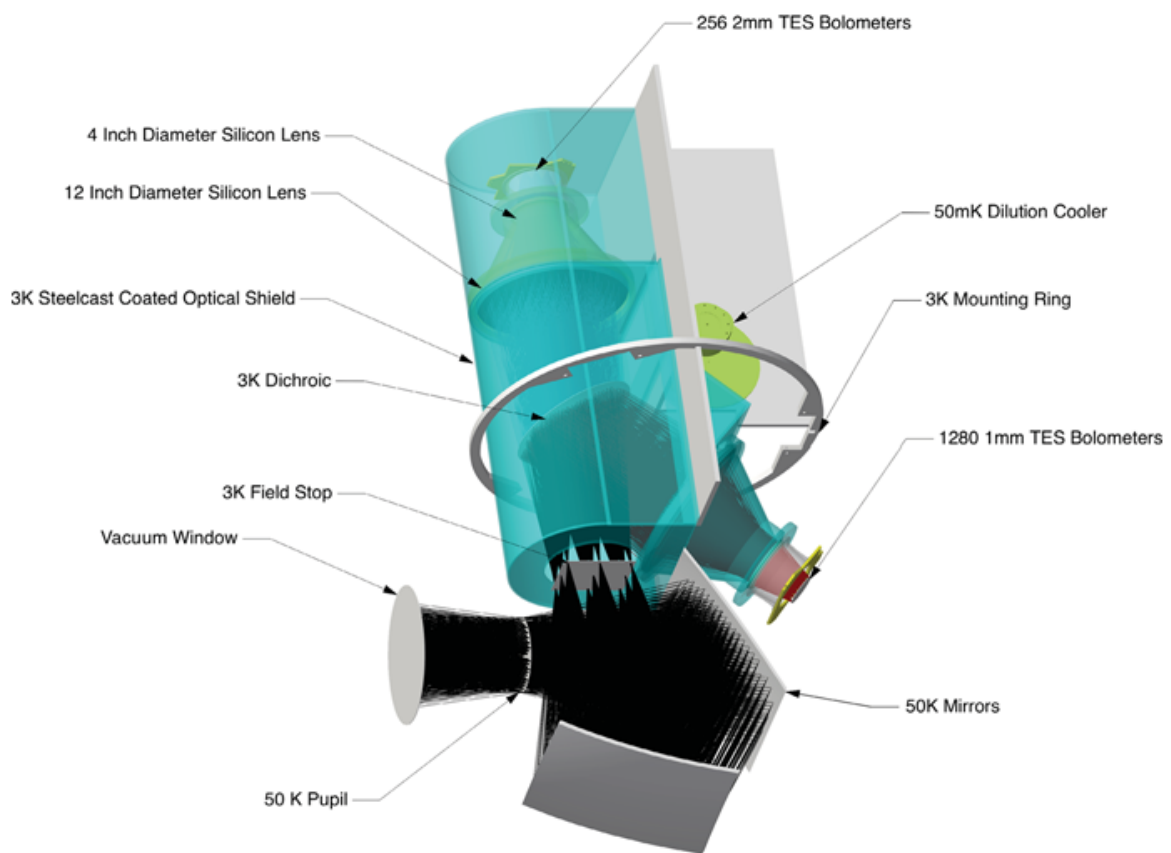

Fig. 4 The GISMO-2 dewar (Color figure online)

\subsubsection{Optics}

In addition to two anti-reflection coated lenses for each color, two curved mirrors at $50 \mathrm{~K}$ yield a $0.8 / 0.9 \lambda / \mathrm{D}(1 / 2 \mathrm{~mm})$ sampling (Fig. 4) in this crossed-Dragone optical design. The sampling is intended to optimize the efficiency of GISMO-2 for simultaneous two color large area blank sky surveys, without compromising the source signal-to-noise ratio and the achievable very near diffraction limited angular resolution [8].

The beam passes through a series of filters at both 77 and $3 \mathrm{~K}$ (Thermal Blocker/ Edge defining filter/ Thermal Blocker) before reaching the dichroic. The dichroic will split the beam into the two colors $(1.2 / 2 \mathrm{~mm})$ and a corresponding band pass filter will be mounted on the windows of the each of the detector packages. The filters and dichroic used in the camera will be provided by Cardiff University.

\subsubsection{Software}

The instrument remote control and data acquisition software for GISMO-2 is presently implemented in GISMO, only minor modifications will be required. The warm readout electronics is the MCE (http://e-mode.phas.ubc.ca/mcewiki/index.php/MCE_ Documents) from the University of British Columbia. As for the GISMO camera, for GISMO-2 we will use the CRUSH software package for data reduction (http://www. submm.caltech.edu/ sharc/crush/index.htm). 
Acknowledgments This work was supported through NSF ATI grants 1020981 and 1106284.

\section{References}

1. J.E. Carlstrom et al., The 10 Meter South Pole Telescope. Publ. Astron. Soc. Pac. 123, 568-581 (2011)

2. J.G. Staguhn, C.A. Allen, D.J. Benford, J.A. Chervenak, D.T. Chuss, T.M. Miller, S.H. Moseley, E.J. Wollack, Characterization of TES bolometers used in 2-dimensional backshort-under-grid (BUG) arrays for far-infrared astronomy. Nuclear Instrum. Methods Phys. Res. A 559, 545-547 (2006)

3. J.G. Staguhn, D.J. Benford, C.A. Allen, S.F. Maher, E.H. Sharp, T.J. Ames, R.G. Arendt, D.T. Chuss, E. Dwek, D.J. Fixsen, T.M. Miller, S.H. Moseley, S. Navarro, A. Sievers, E.J. Wollack, Instrument performance of GISMO, a 2 millimeter TES bolometer camera used at the IRAM $30 \mathrm{~m}$ Telescope, vol. 7020E, in SPIE, (2008) p. 3.

4. P.L. Capak, D. Riechers, N.Z. Scoville, C. Carilli, P. Cox, R. Neri, B. Robertson, M. Salvato, E. Schinnerer, L. Yan, G.W. Wilson, M. Yun, F. Civano, M. Elvis, A. Karim, B. Mobasher, J.G. Staguhn, A massive protocluster of galaxies at a redshift of $\mathrm{z} \sim 5.3$. Nature 470, 233-235 (2011)

5. E. Dwek, J.G. Staguhn, R.G. Arendt, P.L. Capak, A. Kovacs, D.J. Benford, D. Fixsen, A. Karim, S. Leclercq, S.F. Maher, S.H. Moseley, E. Schinnerer, E.H. Sharp, Star and dust formation activities in AzTEC-3, a starburst galaxy at $\mathrm{z}=5.3$. Astrophys. J. 738, 36 (2011)

6. C.A. Allen, D.J. Benford, J.A. Chervenak, D.T. Chuss, T.M. Miller, S.H. Moseley, J.G. Staguhn, E.J. Wollack, Backshort-under-grid arrays for infrared astronomy. Nucl. Inst. And Meth. A 559(2), p522 (2006)

7. J.G. Staguhn, A. Kovacs, R.G. Arendt, D.J. Benford, R. Decarli, E. Dwek, D.J. Fixsen, G.C. Hilton, K.D. Irwin, C.A. Jhabvala, A. Karim, S. Leclercq, S.F. Maher, T.M. Miller, S.H. Moseley, E.H. Sharp, F. Walter, E.J. Wollack, The GISMO 2-millimeter deep field in GOODS-N, arXiv:1311.1485 (2013).

8. G. Bernstein, Advanced exposure-time calculations: undersampling, dithering, cosmic rays, astrometry, and ellipticities. PASP 114, 98 (2002) 